\title{
Two response processes in a guessing task
}

\author{
SHUJI MORI \\ Kyoto University, Kyoto, Japan
}

\begin{abstract}
A guessing task was performed in three conditions of feedback sequence. One sequence was random; the other sequences were nonrandom. Each subject made 300 responses per session for four sessions. The frequency distribution of the differences between response/feedback for the immediately preceding trial and the current response was analyzed per session and for the pooled data. The results suggest that there are two response processes operating in this task: a static process and a dynamic process. This model has two advantages. First, it can explain the sequential dependencies in the present study and in Ward and Lockhead's (1971) Study 3. Second, each of the two processes proposed here has a theoretical foundation: the static process is based on the subject's tendency to generate a random sequence with many alternatives; the dynamic process is a modified version of a learning model in a signal detection task (Dorfman, Saslow, \& Simpson, 1975).
\end{abstract}

In an absolute judgment task, the subject's response $\left(R_{n}\right)$ is affected by not only the present stimulus $\left(S_{n}\right)$, but also by the stimuli and the responses for the preceding trials. These effects of the preceding events are called sequential dependencies (or sequential effects). Much research has focused on the sequential dependencies of absolute judgments (Braida \& Durlach, 1972; Helson, 1959; Holland \& Lockhead, 1968; Luce, Green, \& Weber, 1976; Parducci, 1965; Ward, 1972; Ward \& Lockhead, 1970, 1971; Weber, Green, \& Luce, 1977). In contrast to the large number of studies that have been conducted on the perceptual processes that produce sequential dependencies, however, the response processes have not been dealt with very often. The main reason for this seems to be that the close correlation between stimuli and responses makes it difficult to separate the effects of the response processes from those of the perceptual processes. In spite of such difficulty, the importance of the response processes in an absolute judgment task has been confirmed theoretically and experimentally in several studies (Mori, 1986; Treisman, 1985; Treisman \& Williams, 1984; Ward, 1979; Ward \& Lockhead, 1971). The purpose of the present study was to demonstrate experimentally the response processes that produce sequential dependencies in as pure a form as possible and to develop a theory on the response processes.

As has been generally reported (e.g., Holland \& Lockhead, 1968; Luce et al., 1976; Ward, 1972; Ward \& Lockhead, 1970, 1971), $R_{n}$ is most affected by the im-

This article is based on a master's thesis submitted to the Kyoto University. I wish to express my gratitude to Lawrence $M$. Ward for his valuable suggestions. I also thank Gregory R. Lockhead and an anonymous reviewer for their comments on the manuscript. Reprint requests should be sent to Shuji Mori, Department of Psychology, University of British Columbia, 2136 West Mall, Vancouver, British Columbia V6T 1Y7, Canada. mediately preceding stimulus $\left(S_{n-1}\right)$ and response $\left(R_{n-1}\right)$. This has been quantitatively demonstrated through multiple regression analyses by Green, Luce, and Duncan (1977) and Jesteadt, Luce, and Green (1977). Although weaker, but distinctive, effects of earlier responses and stimuli have been reported (e.g., Staddon, King, \& Lockhead, 1980; Treisman, 1985; Treisman \& Williams, 1984), it may be sufficient for this paper to focus on the effect of immediately preceding events, at least as a firstorder approximation.

The best way to separate the effects of response processes from those of perceptual processes is, I think, the guessing task used in Ward and Lockhead's (1971) Study 3, that is, "an absolute judgment without stimulus" task. As can easily be appreciated, the absence of a stimulus forces subjects to make responses using only their own response processes. Ward (1979) also used a guessing task in experiments on magnitude estimation and cross-modality.

Ward and Lockhead (1971) found, in their Study 3, positive dependencies on immediately preceding feedback $\left(F_{n-1}\right)$ without any dependencies on $R_{n-1}$. Ward and Lockhead explained these results in the following manner. They assumed that the less stimulus information the subject gets, the more likely he/she is to make use of information from sources other than $S_{n}$ in order to raise the rate of correct response. In other words, the sequential dependencies on $S_{n-1}$ and $R_{n-1}$ are dependent on the quantity of stimulus information available to the subjects. Accordingly, in a guessing task in which no stimulus is presented, $F_{n-1}$ given on a correct response is used more often than $R_{n-1}$. When feedbacks are presented randomly, the frequency distribution of the differences between successive feedbacks is skewed, with a peak at a difference of 1 category step. So the subjects are learning such a pattern of the feedback sequence in the course of the task and tend to maximize, though imperfectly, with respect to the difference 
between $F_{n-1}$ and $R_{n}$, that is, to make a response whose value is different by 1 step from $F_{n-1}$. Ward and Lockhead supported this model, showing that the frequency distribution of $F_{n-1}-R_{n}$ differences was more skewed than that of $F_{n-1}-F_{n}$ differences.

Wagner and Baird (1981) used a guessing task similar to that used by Ward and Lockhead (1971), which showed not only strong positive dependencies on $F_{n-1}$, as did Ward and Lockhead's (1971) task, but also slight positive dependencies on $R_{n-1}$. In addition, the frequency distributions of both $R_{n-1}-R_{n}$ and $F_{n-1}-R_{n}$ differences peaked at 0 step. Wagner and Baird (1981) proposed a repetition model showing that subjects tended to repeat $F_{n-1}$ and $R_{n-1}$.

The concept of subjective randomness was adduced as the theoretical ground of the repetition model. It was generally concluded from a great number of studies (see Wagenaar, 1972, for a review) that subjects were unlikely to repeat numerals in a random-generation task, and that the sequences they perceived as random were those that had fewer repetitions than the ideal. Wagner and Baird (1981) assumed that subjects would perceive a random feedback sequence as being more repetitive than random, and that they therefore tended to repeat $F_{n-1}$ and $R_{n-1}$ very often.

Note that the two models assume separately two distinct response processes operating in an absolute judgment task: a static process and a dynamic one. According to signal detection theory, all psychophysical tasks, including guessing tasks, are based on a decision-making process (e.g., Green \& Swets, 1966), and this process has been generally assumed to be static (Dorfman, Saslow, \& Simpson, 1975; Thomas, 1975): the subject locates his/her response criterion on a likelihood ratio continuum, and then keeps his/her criterion through the task as long as the experimental situation is unchanged. We can see that the repetition model implicitly follows this assumption: the assumed tendency to repeat $F_{n-1}$ and $R_{n-1}$ would never change regardless of the number of trials on which the subject might guess.

However, some researchers (Dorfman \& Biderman, 1971; Dorfman et al., 1975; Friedman, Carterette, Nakatani, \& Ahumada, 1968; Kac, 1962; Kinchla, 1963) have reported a dynamic decision process in a signal detection task. They made a basic assumption that the subject's behavior in a signal detection task is dynamic rather than static. The subject's criterion varies over trials, affected by preceding stimuli, responses, feedbacks, and their interrelations (Thomas, 1975). From a methodological point of view, a guessing task can be regarded as a special case of a probability learning experiment (Friedman et al., 1968), and a dynamic response process has always been observed in that type of experiment. Therefore, it may be possible to identify the decision-making process operating in a guessing task with a dynamic process. I think the maximization model emphasizes the dynamic aspect of the response processes, since it can easily be predicted from this model that the response ten- dency will follow the frequency distribution of the differences between successive feedbacks.

Although most researchers who assumed the dynamic response processes used asymptotic analyses to show the changes of response tendencies clearly, Ward and Lockhead (1971) used only pooled data of all trials for their analyses and did not present the change of response tendency. Wagner and Baird (1981) also used pooled data only.

To determine whether the subject's response tendencies would change over trials, I conducted a 1,200-trial guessing task for each subject and divided the 1,200 trials into four sessions so that I could analyze each session separately. Furthermore, to make explicit that the predictions of the two models were different, I used three feedback sequences: one random sequence and two nonrandom sequences. In one of the two nonrandom sequences, the peak of the frequency distribution of the $F_{n-1}-F_{n}$ difference occurred at the middle steps (4 and 5), which, following the maximization model, would make the subject's response tendencies change in such a direction as to maximize the pattern of $F_{n-1}-F_{n}$ differences, as in the case of random sequences. In the other sequence, there was no noticeable peak in the frequency distribution of the $F_{n-1}-F_{n}$ difference, which would result in a uniform frequency distribution of the $F_{n-1}-R_{n}$ difference, since maximization might not work in this condition. If the repetition model held, however, we would not be able to observe any change of response tendency in any condition, but the frequencies of the $F_{n-1}-R_{n}$ difference and the $R_{n-1}-R_{n}$ difference at the 0 step (repetition) would be higher than chance level. In the Discussion, I will propose that both response processes operate in a guessing task, producing sequential dependencies on $F_{n-1}$ and $R_{n-1}$.

\section{METHOD}

A 10-category (1-10) judgment task was used with three conditions of feedback sequence. Note that feedback values 1-10 were presented equally often in all conditions. These conditions were:

Small difference (SD). Feedbacks 1-10 were presented randomly, with the result that the frequency distribution of $F_{n-1}-F_{n}$ differences between successive numerals was skewed with a peak at 1 step, as in Ward and Lockhead (1971).

Table 1

Markov Transition Matrix Used to Produce a Feedback Sequence in the UD Condition

\begin{tabular}{rrrrrcccccc}
\hline & \multicolumn{10}{c}{$F_{n+1}$} \\
\cline { 2 - 9 }$F_{n}$ & 1 & 2 & 3 & 4 & 5 & 6 & 7 & 8 & 9 & 10 \\
\hline 1 & & & & .10 & .20 & .20 & .20 & & .10 & .20 \\
2 & & & & & .10 & .20 & .20 & .20 & .20 & .10 \\
3 & & & & & .10 & .10 & .30 & .30 & .20 & \\
4 & .10 & & & & & .10 & .10 & .30 & .20 & .20 \\
5 & .20 & .10 & .10 & & & & .10 & .10 & .20 & .20 \\
6 & .20 & .20 & .10 & .10 & & & & .10 & .10 & .20 \\
7 & .20 & .20 & .30 & .10 & .10 & & & & & .10 \\
8 & & .20 & .30 & .30 & .10 & .10 & & & & \\
9 & .10 & .20 & .20 & .20 & .20 & .10 & & & & \\
10 & .20 & .10 & & .20 & .20 & .20 & .10 & & & \\
\hline
\end{tabular}


Uniform difference (UD). The Markov transition matrix shown in Table 1 was used to make a sequence in which the frequency distribution of $F_{n-1}-F_{n}$ differences was not peaked at any step(s). The resultant distribution was almost uniform, not peaked, except for the downtum at 0 step due to the restriction that frequency values 1-10 be presented equally often.

Large difference (LD). The Markov transition matrix shown in Table 2 was used so that the frequency distribution of the differences was skewed with a peak at 4 and 5 steps. Figure 1 (open dots) shows the frequency distributions of these conditions.

\section{Subjects}

The participants were 9 Kyoto undergraduates enrolled in a specialized psychology course. Three subjects participated in each of three conditions.

\section{Procedure}

The subjects were told that they were participating in a subliminal perception experiment using a horizontal black line that would be displayed on a CRT very briefly and then be followed by a square black mask. At the onset of each trial, a fixation point was presented for $1 \mathrm{sec}$ in the center of the CRT after a brief presentation of the trial number. Although the subjects were told that a horizontal line would be presented immediately after the fixation point disappeared and that they should make a category judgment (1-10 for the shortest to the longest) of its length, in fact, instead of the line, the black square mask alone was presented immediately after the fixation point disappeared. On each trial, the verbal response given by the subject was entered into the microcomputer by the experimenter and presented on the CRT. After that, feedback was presented just below the response on the display.

There was a 20-trial practice before the main experiment. In the practice, the subjects sometimes complained that the stimulus was not clearly visible. Complainers were told that this experiment was a subliminal perception experiment in which judgments made without confirmation still did reflect sensation magnitude, and that they were not to despair but to continue to try to follow instructions. Each subject made 300 responses in each of four sessions. Each session was composed of five blocks (60 trials per block). A short rest was given between blocks if the subject asked for it. All trials were self-paced. The experimental design was similar to those used in Ward (1979) and Ward and Lockhead's (1971) Study 3.

To statistically compare the frequencies of the $F_{n-1}-R_{n}$ differences and the $R_{n-1}-R_{n}$ differences with those of the $F_{n-1}-F_{n}$ differences, I used two-tailed tests, unless otherwise specified.

\section{RESULTS}

The data were collapsed across 3 subjects in each condition, providing 3,600 responses in each condition for analysis.

Table 3 presents average percentages of correct guesses for session data, as well as pooled data. Although the correct guesses are not significantly different from chance level $(10 \%)$ in the SD condition, they increase with sessions in the UD and the LD conditions and are significantly higher than chance level in the third and the fourth sessions.

\section{The Frequency Distributions of the Differences Between $F_{n-1}$ and $R_{n}$ and of the Differences Between Successive Responses}

Figure 1 shows the frequency distributions of $F_{n-1}-R_{n}$ differences for the pooled data of all sessions. All of their
Table 2

Markov Transition Matrix Used to Produce a Feedback Sequence in the LD Condition

\begin{tabular}{rcccccccccc}
\hline \multicolumn{10}{c}{$F_{n+1}$} \\
\hline$F_{n}$ & 1 & 2 & 3 & 4 & 5 & 6 & 7 & 8 & 9 & 10 \\
\hline 1 & & & & & & & & .15 & .35 & .50 \\
2 & & & & & & .05 & .10 & .20 & .30 & .35 \\
3 & & & & & .05 & .10 & .20 & .30 & .20 & .15 \\
4 & & & & .10 & .10 & .20 & .30 & .20 & .10 & \\
5 & & & .05 & .10 & .20 & .30 & .20 & .10 & .05 & \\
6 & & .05 & .10 & .20 & .30 & .20 & .10 & .05 & & \\
7 & & .10 & .20 & .30 & .20 & .10 & .10 & & & \\
8 & .15 & .20 & .30 & .20 & .10 & .05 & & & & \\
9 & .35 & .30 & .20 & .10 & .05 & & & & & \\
10 & .50 & .35 & .15 & & & & & & & \\
\hline
\end{tabular}

shapes are clearly different from those of the $F_{n-1}-F_{n}$ differences of each condition. The result of the SD condition closely resembles that of Ward and Lockhead's (1971) Study 3. The frequency of the difference at 1 step is the highest in the distribution, and is significantly higher than that of the $F_{n-1}-F_{n}$ difference $(Z=4.26, p<.01)$. In the LD condition, although the frequency distribution of the $F_{n-1}-R_{n}$ differences peaks at 3 steps, close to the 4-step peak of the $F_{n-1}-F_{n}$ differences, the frequency of the difference at the 4-step point is significantly lower than that of the $F_{n-1}-F_{n}$ differences $(Z=23.3, p<.01)$. In the UD condition, the frequency distribution of $F_{n-1}-R_{n}$ differences peaks at small steps ( 1 and 2 ), and the frequencies at 1 and 2 steps are significantly higher than those of the $F_{n-1}-F_{n}$ difference ( $Z s=9.30$ and 8.64 for 1 and 2 steps, respectively; both $p s<.01$ ).

Figure 2 shows the frequency distributions of the $F_{n-1}-R_{n}$ differences for the session data of each condition. All distributions in each condition are different from those of the $F_{n-1}-F_{n}$ differences. In the SD condition, all distributions peak at 1 step, and the frequencies at 1 step are significantly higher for the third and fourth sessions than are those of the $F_{n-1}-F_{n}$ difference $(Z=3.26$, $p<.01$, for the third session, and $Z=2.21, p<.05$, for the fourth session). In the UD condition, the peak shifts from 1 step in the first and second sessions to 2 steps in the third and the fourth sessions, and the frequencies at 1 and 2 steps are significantly higher than those of the

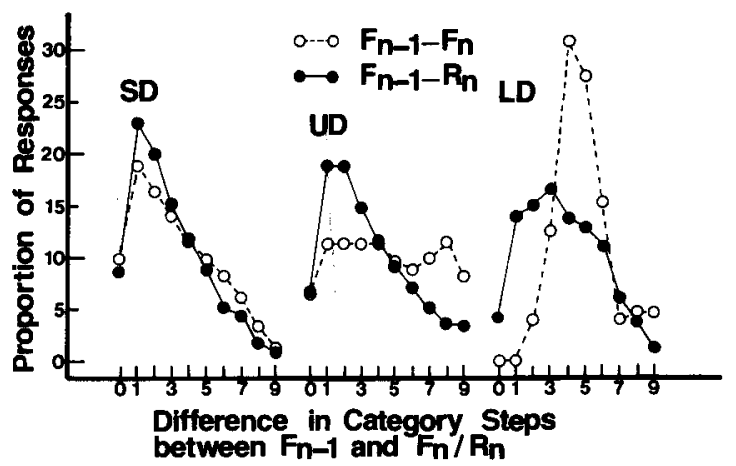

Figure 1. The frequency distributions of the differences in category steps between $F_{n-1}$ and $F_{n}$, and the differences between $F_{n-1}$ and $R_{n}$ for the pooled data of three conditions. 
Table 3

Proportions of Correct Guesses and Comparisons with Chance Level (10\%), Using Two-Tailed Tests

\begin{tabular}{|c|c|c|c|c|c|c|c|c|c|c|}
\hline \multirow[b]{3}{*}{ Condition } & \multicolumn{10}{|c|}{ Sessions } \\
\hline & \multicolumn{2}{|l|}{1} & \multicolumn{2}{|l|}{2} & \multicolumn{2}{|l|}{3} & \multicolumn{2}{|l|}{4} & \multicolumn{2}{|c|}{ Pooled } \\
\hline & Prop. (\%) & $Z$ & Prop. $(\%)$ & $Z$ & Prop. $(\%)$ & $Z$ & Prop. (\%) & $Z$ & Prop. (\%) & $Z$ \\
\hline SD & 11.4 & .99 & 10.6 & .39 & 9.4 & .40 & 10.7 & 1.28 & 10.5 & .74 \\
\hline UD & 10.4 & .31 & 10.9 & .62 & 12.9 & 1.93 & 13.7 & $2.41^{*}$ & 12.0 & $2.68 *$ \\
\hline LD & 11.1 & .77 & 10.0 & .00 & 13.0 & $1.99+$ & 17.8 & $4.77^{*}$ & 13.0 & $3.95^{*}$ \\
\hline
\end{tabular}

$F_{n-1}-F_{n}$ difference in all sessions $(p<.01$ in all cases). In addition, the frequencies at large steps $(7,8$, and 9$)$ increase with sessions. In the LD condition, the peak shifts from 1 to the middle steps $(3,4$, and 5$)$, although the frequencies at 4 and 5 steps are significantly lower than those between successive feedbacks, even in the fourth session (Zs $=5.88$ and 4.90 , for 4 and 5 steps, respectively; both ps $<.01)$.

(a)

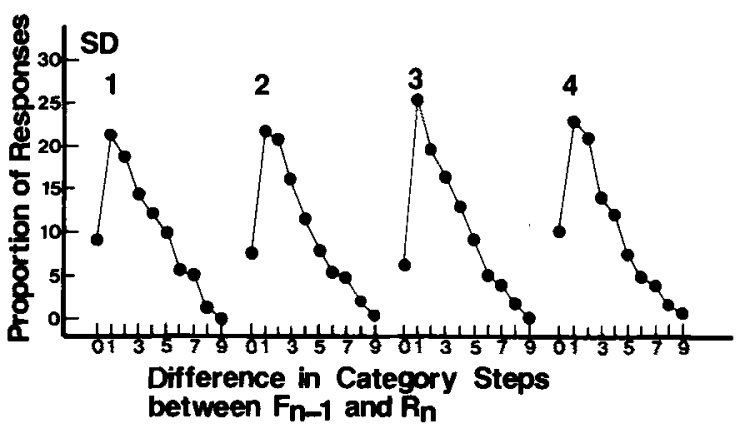

(b)

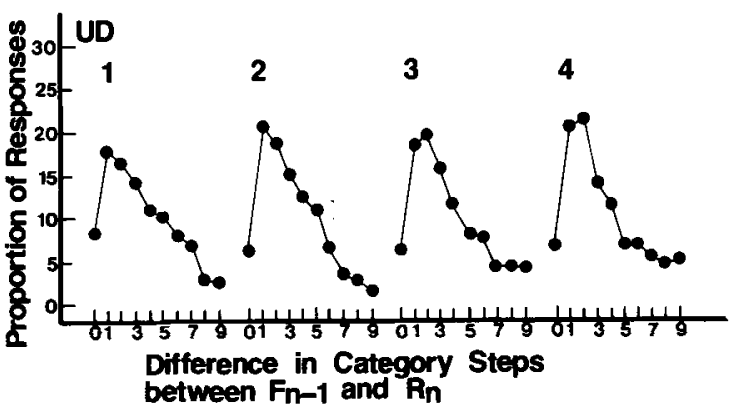

(c)

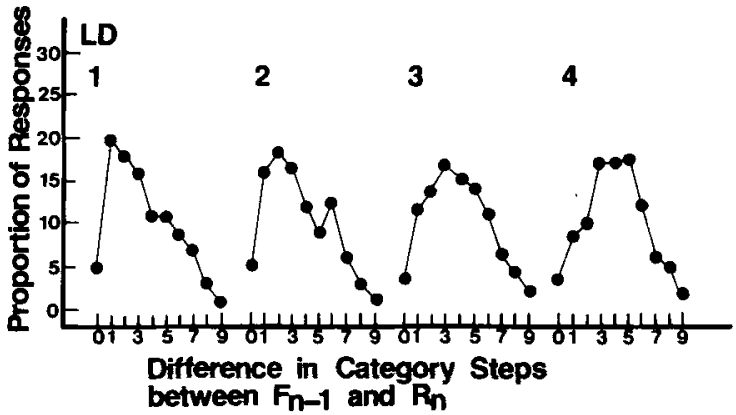

Figure 2. The frequency distributions of the differences in category steps between $F_{n-1}$ and $R_{n}$ for the session data of three conditions.
As mentioned above, it is predicted from the repetition model of Wagner and Baird (1981) that the frequency of the $F_{n-1}-R_{n}$ difference at 0 step as well as that of the $R_{n-1}-R_{n}$ difference at 0 step should be higher than chance level in all conditions. Table 4 presents the results of a comparison of the frequencies of the $F_{n-1}-R_{n}$ difference at 0 step with chance level $(10 \%)$ for the pooled data and the session data of all conditions. The pooled data show that the frequency of the $\mathbf{0}$-step difference is significantly less than chance in all conditions. Regarding the session data, except for the third session, the SD condition has no session that is significantly different from chance level. However, most sessions of the UD and the LD conditions are significantly lower than chance level.

Figure 3 shows the frequency distributions of the $R_{n-1}-R_{n}$ differences for the pooled data. All of them differ from Wagner and Baird's (1981) prediction. In all distributions, the frequency at 1 step is the highest and the frequency at 0 step is lower than chance level.

Figure 4 shows the frequency distributions of the $R_{n-1}-R_{n}$ differences for the session data. Almost all of them peak at 1 step. In the UD condition, however, the frequencies at large steps $(7,8$, and 9$)$ increase with sessions. Only the distribution of the fourth session in the LD condition peaks at 4 steps.

Table 5 presents the results of the comparison of the frequencies of the $R_{n-1}-R_{n}$ difference at 0 step with chance level, for the session data as well as the pooled data of all conditions. Except for the third session of the SD condition, the 0-step difference has significantly lower frequency than chance level for the session data of all conditions.

\section{Sequential Dependencies}

Figure 5 shows the feedback and response analyses that reveal sequential dependencies on $F_{n-1}$ and on $R_{n-1}$ for the pooled data. This method of analysis was introduced by Holland and Lockhead (1968) and is generally used to show the sequential dependencies of absolute judgments. Each point in the figures represents the average response on Trial $n$ as a function of feedback or response value on Trial $n-1$ (Wagner \& Baird, 1981, and Ward \& Lockhead, 1971, extended the analyses to a greater number of preceding trials). The mean response on Trial $n$ is calculated separately for pairs of feedback or response values on Trial $n-1$. 
Table 4

Proportions of 0-step Differences Between $F_{n-1}$ and $R_{n}$ and Comparisons with Chance Level (10\%), Using Two-Tailed Tests

\begin{tabular}{|c|c|c|c|c|c|c|c|c|c|c|}
\hline \multirow[b]{3}{*}{ Condition } & \multicolumn{10}{|c|}{ Sessions } \\
\hline & \multicolumn{2}{|l|}{1} & \multicolumn{2}{|l|}{2} & \multicolumn{2}{|l|}{3} & \multicolumn{2}{|l|}{4} & \multicolumn{2}{|c|}{ Pooled } \\
\hline & Prop. (\%) & $Z$ & Prop. (\%) & $Z$ & Prop. (\%) & $Z$ & Prop. (\%) & $Z$ & Prop. (\%) & $Z$ \\
\hline SD & 9.3 & .17 & 7.8 & 1.34 & 6.3 & $2.81 *$ & 10.2 & .46 & 8.4 & $2.27 \dagger$ \\
\hline UD & 8.3 & 1.20 & 6.3 & $2.81^{*}$ & 6.4 & $2.72 *$ & 6.5 & $2.62 *$ & 6.9 & $4.65^{*}$ \\
\hline LD & 4.7 & $4.21 *$ & 5.3 & $3.69 *$ & 3.5 & $5.41^{*}$ & 3.6 & $5.30 *$ & 4.3 & $9.27 *$ \\
\hline
\end{tabular}

The results of the SD condition agree quite well with Wagner and Baird (1981), and Ward and Lockhead's (1971) Study 3 in showing strong positive dependencies on $F_{n-1}$. However, there are marked negative dependencies on $F_{n-1}$ in the UD and LD conditions. The tendencies revealed by the response analyses are the same as, though a little weaker than, those revealed by the feedback analyses in each condition.

Figure 6 shows the feedback and response analyses for the session data. In the SD condition, positive dependencies on $F_{n-1}$ are shown over sessions. Conversely, the positive dependencies on $R_{n-1}$ are shown to decrease slightly with sessions. In the UD and LD conditions, although feedback analyses do not show any dependencies in the first session, the negative dependencies on $F_{n-1}$ become strong. Response analyses in both conditions show the same tendencies as do the feedback analyses, but the dependencies are weaker than those on $F_{n-1}$, as observed for pooled data.

Partial correlation coefficients were calculated between $R_{n}$ and $F_{n-1}$ (partialing out $R_{n-1}$ ) and between $R_{n}$ and $R_{n-1}$ (partialing out $F_{n-1}$ ) to give a quantitative expression to the response and the feedback analyses. For the SD condition, these are the same as correlation coefficients, since $F_{n-1}$ and $R_{n-1}$ are uncorrelated. For the UD and LD conditions, however, partial correlation coefficients are thought to be better estimates, since, because they are nonrandom, $F_{n-1}$ and $R_{n-1}$ are correlated, as indicated by high percentages of correct response in Table 1. Partial correlation coefficients, which are presented in Tables 6 and 7 , reveal the same tendencies mentioned above.

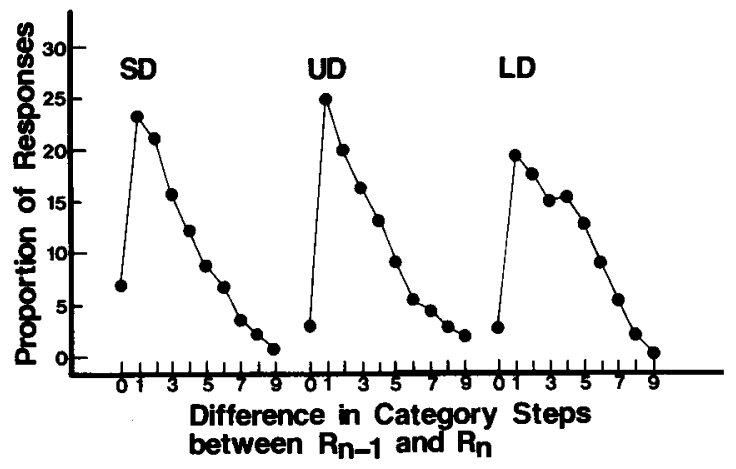

Figure 3. The frequency distributions of the differences in category steps between $R_{n-1}$ and $R_{n}$ for the pooled data of three conditions.

\section{DISCUSSION}

I propose that two different response processes operate in a guessing task, producing sequential dependencies on $F_{n-1}$ and on $R_{n-1}$. One of these processes is static, which shows response tendencies like those observed in 10-numeral random-sequence-generation experiments (Chapanis, 1953; Jenkins \& Cunningham, 1949; Rath, 1966), resulting in constant and positive dependencies on $F_{n-1}$ and $R_{n-1}$. The other process is dynamic, which changes the dependencies on $F_{n-1}$ in such a way as to raise the proportion of correct responses. The basic assumption of this model is that a subject uses the best information available in his task in order to raise the proportion of correct responses, as proposed by Ward and Lockhead (1971).

\section{Static Process}

For a static process, Wagner and Baird (1981) proposed the repetition model, in which subjects tended to repeat $F_{n-1}$ and/or $R_{n-1}$. It is predicted from the repetition model that the frequencies of the $F_{n-1}-R_{n}$ difference and the $R_{n-1}-R_{n}$ difference at 0 step should be significantly higher than chance level in all conditions. The present data, however, show the opposite results: whether they are pooled or session data, most show significantly lower frequencies than chance level (Tables 4 and 5). In addition, we can also see that the response tendency to make a guess is most often different in 1 step from $F_{n-1}$ or $R_{n-1}$. In the SD condition, these tendencies are obvious not only for pooled data (Figure 1) but also for session data, even those for the first session (Figure 2a). They are also observed in the first and the second sessions of both the UD and LD conditions (Figure $2 b$ and $2 c$ ).

For a static process, I propose that the subject avoids the repetition of $F_{n-1}$ and $R_{n-1}$ and makes a guess most often 1 step away from $F_{n-1}$ and $R_{n-1}$. I also adduce a subject's random-generation behavior as the theoretical basis for the static process. It is true that the static process proposed here directly contradicts the assumption of the repetition model, so that as its theoretical foundation I cannot introduce the concept of subjective randomness, upon which the repetition model is based. However, that concept was based mainly on the results of randomgeneration experiments using two alternatives, and the results of random-generation experiments vary with the 
(a)

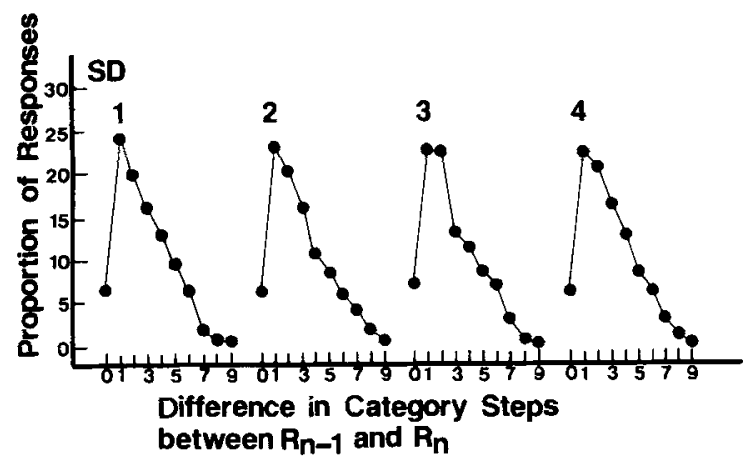

(b)

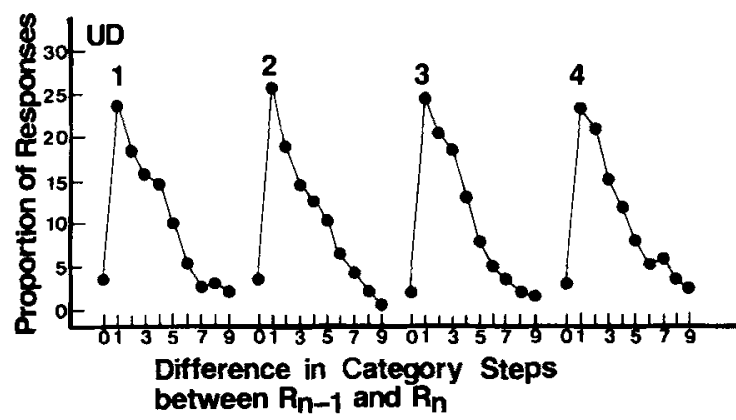

(c)

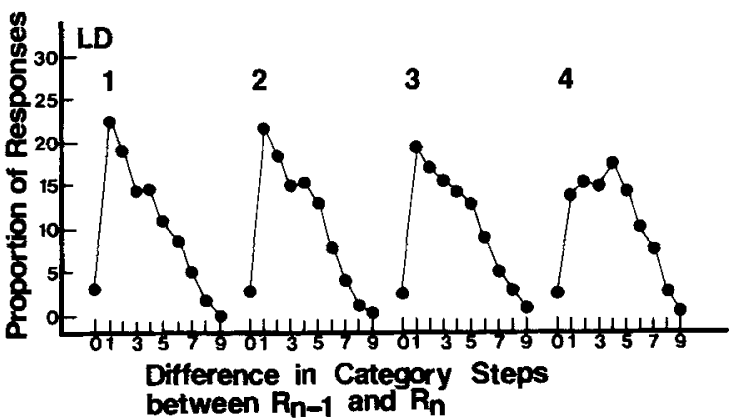

Figure 4. The frequency distributions of the differences in category steps between $R_{n-1}$ and $R_{n}$ for the session data of three conditions.

number of alternatives (Wagenaar, 1972). In fact, the results of random-generation experiments using 10 numerals (Chapanis, 1953; Jenkins \& Cunningham, 1949; Rath, 1966) have shown not only few repetitions but also many 1-step differences, which agrees quite well with the results of both the present study and Ward and Lockhead's (1971). Therefore, I propose that the static process is based on the subject's tendency to generate a number from many alternatives without an informational cue. This proposal is also supported by the fact that even in the UD and LD conditions, in which a nonrandom sequence is used as feedback, the same tendencies can be observed even before a subject has become accustomed to the task. Accordingly, although I find the pooled data of the SD condition to closely match those of Ward and Lockhead's (1971) Study 3, which supported their maximization model, both the pooled and the session data, especially those of the beginning sessions of the UD and LD conditions, can be explained by the static process proposed here.

The contradiction between the results obtained by Wagner and Baird (1981) and those obtained in the present study most probably arises from a procedural difference. Wagner and Baird told subjects that they were in an ESP experiment, and asked them to guess which of the numerals 1-9 had been chosen. Their subjects would try very hard to make only correct guesses, and they would choose a strategy for the purpose, such as repeating the same numeral. On the other hand, the subjects in the present study were asked to perceive a "stimulus" and respond to it. The reason I chose, in the present study, to use a guessing task, as had Ward and Lockhead (1971) and Wagner and Baird (1981), was to be able to study response processes in an absolute judgment task, and I think the procedure of the present study suits that purpose better than did Wagner and Baird's.

The static process produces positive sequential dependencies on $F_{n-1}$ and on $R_{n-1}$, as explained by Ward and Lockhead (1971). In choosing a response, the subjects have 10 alternatives: 1 to 10 . Since they cannot get information from $S_{n}$, because it is in fact not presented, their choice of $R_{n}$ depends greatly upon $F_{n-1}$ and $R_{n-1}$. As stated above, the static process makes them choose a response 1 step away from $F_{n-1}$ or $R_{n-1}$ most often. If $F_{n-1}$ is 2 , for example, they choose 1 or 3 more often than any other alternatives, even if $F_{n}$ is 9 or 10 ; if $F_{n-1}$ is 9 , then 8 or 10 is chosen most often. This means that $R_{n}$ would be lower when $F_{n-1}$ was lower and higher when $F_{n-1}$ was higher. The same thing would happen in the case of $R_{n-1}$. Accordingly, there are positive dependencies on $F_{n-1}$ and $R_{n-1}$ for the pooled data as well as the session data in the SD condition (Figures 5 and 6a).

As Figure 6a shows, however, the positive dependencies on $R_{n-1}$ weaken in the third and the fourth sessions

Table 5

Proportions of 0-step Differences Between $R_{n-1}$ and $R_{n}$ and Comparisons with Chance Level (10\%), Using Two-Tailed Tests

\begin{tabular}{|c|c|c|c|c|c|c|c|c|c|c|}
\hline \multirow[b]{3}{*}{ Condition } & \multicolumn{10}{|c|}{ Sessions } \\
\hline & \multicolumn{2}{|l|}{1} & \multicolumn{2}{|l|}{2} & \multicolumn{2}{|l|}{3} & \multicolumn{2}{|l|}{4} & \multicolumn{2}{|c|}{ Pooled } \\
\hline & Prop. (\%) & $Z$ & Prop. (\%) & $Z$ & Prop. (\%) & $\boldsymbol{Z}$ & Prop. (\%) & $Z$ & Prop. (\%) & $\mathcal{Z}$ \\
\hline $\begin{array}{l}\text { SD } \\
\text { UD } \\
\text { LD }\end{array}$ & $\begin{array}{l}6.6 \\
3.5 \\
3.0\end{array}$ & $\begin{array}{l}2.53 \dagger \\
5.41^{*} \\
6.00^{*}\end{array}$ & $\begin{array}{l}6.6 \\
3.4 \\
2.5\end{array}$ & $\begin{array}{l}2.53 \dagger \\
5.53^{*} \\
6.49^{*}\end{array}$ & $\begin{array}{l}7.6 \\
2.0 \\
2.6\end{array}$ & $\begin{array}{l}1.72 \\
7.12^{*} \\
6.36^{*}\end{array}$ & $\begin{array}{l}6.3 \\
2.8 \\
2.6\end{array}$ & $\begin{array}{l}2.81^{*} \\
6.32^{*} \\
6.36^{*}\end{array}$ & $\begin{array}{l}6.8 \\
2.9 \\
2.7\end{array}$ & $\begin{array}{r}4.79^{*} \\
12.06^{*} \\
12.61^{*}\end{array}$ \\
\hline
\end{tabular}

${ }^{*} p<.01 . \quad \dagger p<.05$. 


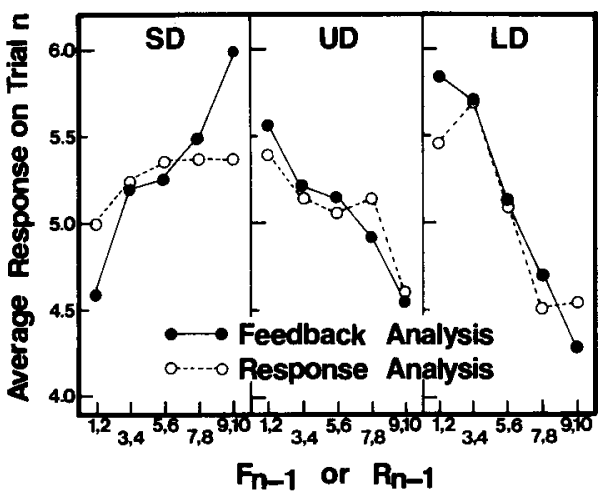

Figure 5. Average responses on Trial $\boldsymbol{n}$ as a function of feedback or response value on Trial $n-1$ for the pooled data of three conditions.

of the SD condition. This is also evident in the top row of Table 2. To explain this, I adopt Ward and Lockhead's (1971) assumption that a subject uses the best information available to raise the proportion of correct responses. In the beginning part of the task, the subjects used $\boldsymbol{R}_{n-1}$ as well as $F_{n-1}$ for such information. However, as they got accustomed to the task, they found $R_{n-1}$ would not give them useful information. Accordingly, they did not use $R_{n-1}$ in the third and fourth sessions as often as they had in the first and second sessions, resulting in the weaker dependencies on $\boldsymbol{R}_{n-1}$.

\section{Dynamic Process}

The static process proposed here never changes-at least systematically-the response tendencies that are shown as the frequency distributions of the $F_{n-1}-R_{n}$ or the $R_{n-1}-R_{n}$ differences. Nevertheless, we can see the peakshifting of the distribution of $F_{n-1}-R_{n}$ differences with sessions in the UD and LD conditions. In the LD condition, the peak is at 1 step in the first session and then gradually shifts to 4 and 5 steps (Figure $2 \mathrm{c}$ ). This pattern can also be seen in the UD condition; that is, the peak is at 1 step in the first and the second sessions and then shifts to 2 steps (Figure 2b). Therefore, it seems that some dynamic process is operating in the UD and LD conditions. However, I think the dynamic process is unlike the maximization model. Following the maximization model, the middle-step (4 and 5) frequencies of the $F_{n-1}-R_{n}$ difference in the LD condition should be higher than those of the $F_{n-1}-F_{n}$ difference. However, they are, in fact, lower than those of the $F_{n-1}-F_{n}$ differences. In addition, the frequencies at large steps $(7,8$, and 9$)$ gradually increase with sessions in not only the LD condition but also the UD condition, which would not be observed if the subjects had guessed as predicted by the maximization model. Rather, this response tendency is classified as probability matching according to probability learning theory (Estes, 1964), that is, the subjects seem to be trying to match the pattern of $F_{n-1}-R_{n}$ differences with that of
$F_{n-1}-F_{n}$ differences. In other words, the shape of the frequency distribution of $F_{n-1}-R_{n}$ difference is changing toward that of $F_{n-1}-F_{n}$ differences. So the peak shifts and the frequency increases of the distribution of $F_{n-1}-F_{n}$ differences occur at large steps for the session data in the UD and LD conditions. As the subjects had not yet reached perfect matching even in the fourth session, the

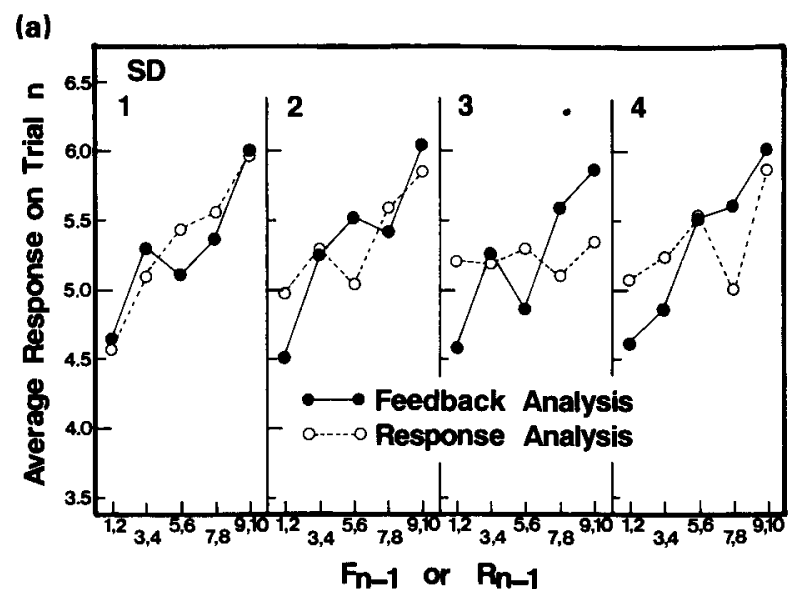

(b)

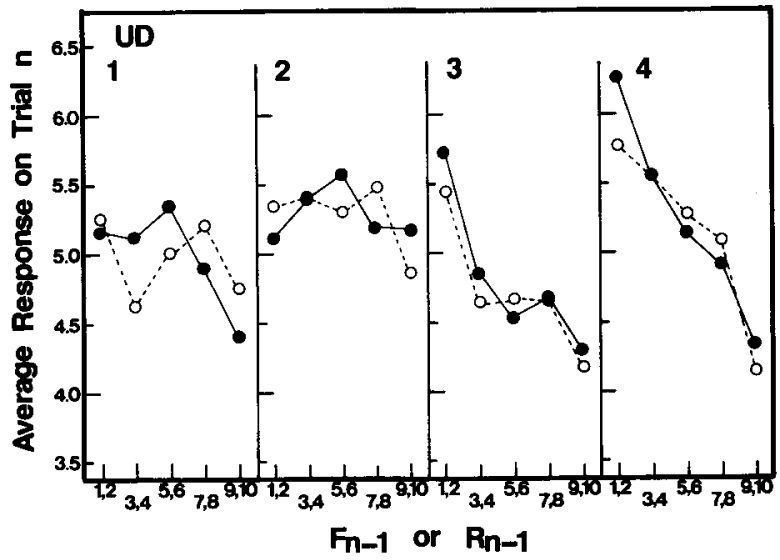

(c)

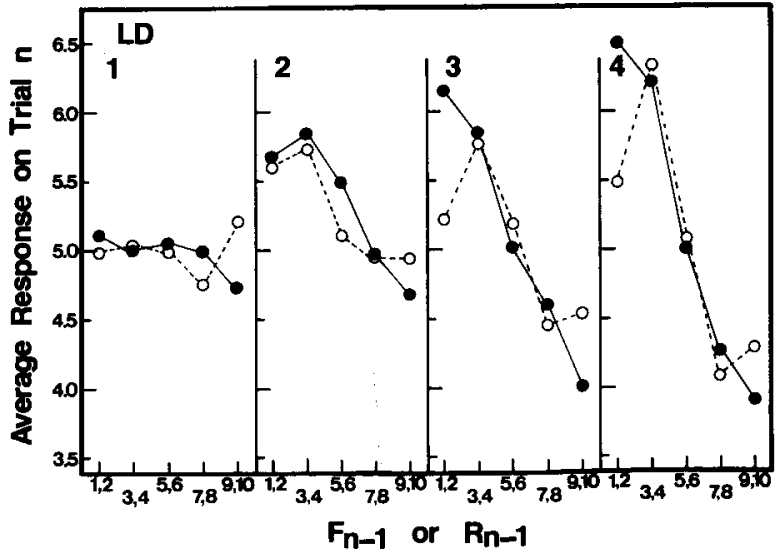

Figure 6. Average responses on Trial $\boldsymbol{n}$ as a function of feedback or response value on Trial $n-1$ for the session data of three conditions. 
Table 6

Partial Correlation Coefricients Between $F_{n-1}$ and $R_{n}$ (Partialing Out $R_{n-1}$ ) for Pooled Data of Three Conditions

\begin{tabular}{cccccc}
\hline & \multicolumn{5}{c}{ Sessions } \\
\cline { 2 - 6 } Condition & 1 & 2 & 3 & 4 & Pooled \\
\hline SD & $.155^{*}$ & $.171^{*}$ & $.163^{*}$ & $.200^{*}$ & $.173^{*}$ \\
UD & $-.08 \dagger^{*}$ & -.032 & $-.107^{*}$ & $-.151^{*}$ & $-.095^{*}$ \\
LD & -.044 & $-.101^{*}$ & $-.258^{*}$ & $-.266^{*}$ & $-.169^{*}$ \\
\hline${ }^{*} p<.01 . \quad \dagger p<.05$. & & & &
\end{tabular}

Table 7

Partial Correlation Coeficicients Between $R_{n-1}$ and $\boldsymbol{R}_{n}$ (Partialing Out $\boldsymbol{F}_{n-1}$ ) for Pooled Data of Three Conditions

\begin{tabular}{cccccr}
\hline & \multicolumn{5}{c}{ Session } \\
\cline { 2 - 6 } Condition & 1 & 2 & 3 & 4 & Pooled \\
\hline SD & $.160^{*}$ & $.088^{*}$ & .005 & .043 & $.065^{*}$ \\
UD & $-.00 \dagger^{*}$ & -.048 & $-.100^{*}$ & $-.168^{*}$ & $-.057^{*}$ \\
LD & $-.033^{*}$ & $-.114^{*}$ & $-.113^{*}$ & $-.184^{*}$ & $-.114^{*}$ \\
\hline
\end{tabular}

${ }^{*} p<.01 . \quad \dagger p<.05$

frequencies of the $F_{n-1}-R_{n}$ differences at a peak point are different from those of the $F_{n-1}-F_{n}$ differences in both conditions.

For a dynamic process operating in the UD and LD conditions, I propose that there is a response tendency for the subject to guess in such a way as to match the frequency distribution of the $F_{n-1}-R_{n}$ differences with that of the $F_{n-1}-F_{n}$ differences. At the beginning of the task, before the subject has become accustomed to it, his/her guess is based on the static process as it is assumed to operate in the SD condition. Unlike the SD condition, however, the UD and LD conditions give subjects a nonrandom sequence as feedback (Tables 1 and 2), so they may easily recognize the pattern of the differences between successive feedbacks given as "correct responses." As they get accustomed to the task, they are likely to guess in such a way as to match the pattern of $F_{n-1}-R_{n}$ differences with that of $F_{n-1}-F_{n}$ differences in order to raise the proportion of correct responses. To support the validity of the dynamic processes in a signal detection task, I can adduce a learning model for the response process in a signal detection task (Dorfman \& Biderman, 1971; Dorfman et al., 1975; Friedman et al., 1968; Ward, 1973; Ward, Livingston, \& Li, 1988). The learning model is based on the assumption that the response process operating in a signal detection task is dynamic. According to this assumption, Dorfman et al. (1975) made an asymptotic analysis of Dorfman and Biderman's (1971) data and showed that the proportion of responses was approaching an asymptote of the proportion of stimuli presented, that is, probability matching. Since the assumption and experimental evidence of the learning model agree quite well with those of the dynamic process proposed here, it seems natural that the dynamic process in a guessing task is a modified version of the learning model.

The response tendencies based on the dynamic process result in negative dependencies on $F_{n-1}$ in the UD and LD conditions (Figure $6 \mathrm{~b}$ and $6 \mathrm{c}$ and Table 6). Compared with the SD condition, there are relatively high frequencies of $F_{n-1}-F_{n}$ differences at steps beyond the middle ones (4 and 5) and a relatively lower frequency at 1 step in both conditions. Therefore, as the subjects are matching the pattern of $F_{n-1}-R_{n}$ differences with that of $F_{n-1}-F_{n}$ differences, they are making a guess more than 4 or 5 steps away from $F_{n-1}$ and avoiding choosing responses that are 1 step different from $F_{n-1}$. Since the subjects' response is limited to 10 alternatives, 1 to 10 , such response tendencies would result in negative dependencies on $F_{n-1}$.

There are also negative dependencies on $R_{n-1}$ in the UD and LD conditions, which are weaker than those on $F_{n-1}$ for the session data as well as for the pooled data (Table 7). Referring back to the explanation given in the Static Process section to explain the weaker dependencies on $R_{n-1}$ for the third and the fourth sessions in the SD condition, I again adopt Ward and Lockhead's (1971) assumption that a subject uses the best information available to raise his/her proportion of correct responses. In a guessing task, the subject would use $F_{n-1}$, rather than $R_{n-1}$, for such information. However, the fact that the average percentage of correct guesses is increasing with sessions (Table 3 ) suggests that the correlation of feedback and response should be increasing in both conditions. Accordingly, it is concluded that negative dependencies on $R_{n-1}$ should follow negative dependencies on $F_{n-1}$. This conclusion is supported by the result that the shape changing of the frequency distribution of $R_{n-1}-R_{n}$ differences with sessions is similar to, but slower than, that of $F_{n-1}-R_{n}$ differences (Figure 4).

It is noteworthy that the frequency of the $F_{n-1}-R_{n}$ difference at 1 step is not higher than chance level for the first session in the UD and LD conditions (Figure $2 b$ and $2 c$ ). This suggests that the dynamic process would operate even at the beginning of both conditions. I assume that the static 
process and the dynamic process would operate simultaneously at the beginning of both conditions. Since the positive dependencies due to the static process and the negative dependencies due to the dynamic process cancel each other, marked dependencies cannot be observed during the beginning sessions.

I have explained successfully the present results by assuming two response processes. As far as the results of the SD condition are concerned, however, they can also be explained by Ward and Lockhead's (1971) maximization model, and the present results do not provide a way to decide which of the two models holds in the SD condition. Studies of such models remain to be done.

\section{REFERENCES}

BraIDA, L. D., \& DuRLACH, N. I. (1972). Intensity perception: II. Resolution in one interval paradigms. Journal of Acoustical Society of America, 51, 483-502.

Chapanis, A. (1953). Random-number guessing behavior. American Psychologist, 18, 332.

Dorfman, D. D., Biderman, M. (1971). A learning model for a continuum of sensory states. Journal of Mathematical Psychology, 8, 264-284.

Dorfman, D. D., SAsLow, C. F., \& Simpson, J. C. (1975). Learning models for a continuum of sensory states reexamined. Journal of Mathematical Psychology, 12, 178-211.

Estes, W. K. (1964). Probability learning. In A. W. Melton (Ed.), Categories of human learning (pp. 89-128). New York; Academic Press.

Friedman, M. P., Carterette, E. C., Nakatani, L., \& Ahumada, A. (1968). Comparison of some learning models for response bias in signal detection. Perception \& Psychophysics, 3, 5-11.

Green, D. M., LuCE, R. D., DunCaN, J. E. (1977). Variability and sequential effects in magnitude production and estimation of auditory intensity. Perception \& Psychophysics, 22, 450-460.

GREEN, D. M., \& SWETS, J. A. (1966). Signal detection theory and psychophysics. New York: Wiley.

Helson, H. (1959). Adaptation level theory. In S. Koch (Ed.), Psychology: A study of a science (Vol. 1, pp. 565-621). New York: McGraw-Hill.

Holland, M. K., Lockhead, G. R. (1968). Sequential effects in absolute judgments of loudness. Perception \& Psychophysics, 3, 409-414.

Jenkins, W. O., \&unningham, L. M. (1949). The guessing-sequence hypotheses, the 'spread of effect,' and number-guessing habits. Joumal of Experimental Psychology, 53, 297-303.

JesteadT, W., LuCE, R. D., * GREEN, D. M. (1977). Sequential effects in judgments of loudness. Joumal of Experimental Psychology: Human Perception \& Performance, 3, 92-104.

$\mathrm{KAC}, \mathrm{M}$. (1962). A note on learning signal detection. IRE Transactions of Information Theory, IT-8, 126-128.
Kinchla, R. A. (1963). A learning factor in visual discrimination. In R. Atkinson (Ed.), Studies in mathematical psychology (pp. 233-249). Stanford: Stanford University Press.

LuCe, R. D., GreEN, D. M. , \& Weber, D. L. (1976). Attention bands in absolute identification. Perception \& Psychophysics, 20, 49-54.

Mor, S. (1986). Sequential dependencies of psychophysical judgments. Unpublished master's thesis, Kyoto University, Kyoto.

Parducci, A. (1965). Category judgment: A range-frequency model. Psychological Review, 72, 407-418.

Rath, G. J. (1966). Randomization by humans. American Journal of Psychology, 79, 97-103.

Staddon, J. E. R., King, M., \& Lockhead, G. R. (1980). On sequential effects in absolute judgment experiments. Journal of Experimental Psychology: Human Perception \& Performance, 6, 290-301.

Thомаs, E. A. C. (1975). Criterion adjustment and probability matching. Perception \& Psychophysics, 18, 158-162.

Treisman, M. (1985). The magical number seven and some other features of category scaling: Properties of a model for absolute judgment. Journal of Mathematical Psychology, 29, 175-230.

Treisman, M., \& Williams, T. C. (1984). A theory of criterion setting with an application to sequential dependencies. Psychological Review, 91, 68-111.

WAGENAAR, W. A. (1972). Generation of random sequence by human subjects: A critical survey of literature. Psychological Bulletin, 77, 65-72.

WAGNER, M., \& BAIRD, J. C. (1981). A quantitative analysis of sequential effects with numeric stimuli. Perception \& Psychophysics, 29, 359-364.

WARD, L. M. (1972). Category judgments of loudness in the absence of an experimenter-induced identification function: Sequential effects and power function fit. Journal of Experimental Psychology, 94, 179-184.

WARD, L. M. (1973). Use of Markov-encoded sequential information in numerical signal detection. Perception \& Psychophysics, 14, 337-342.

WARD, L. M. (1979). Stimulus information and sequential dependencies in magnitude estimation and cross-modality matching. Journal of Experimental Psychology: Human Perception \& Performance, 5, 444-459.

WARD, L. M., Livingston, J. W., JR., \& LI, J. (1988). On probabilistic categorization: The Markovian observer. Perception \& Psychophysics, 43, 125-136.

WARD, L. M., \& LOCKHEAD, G. R. (1970). Sequential effects and memory in category judgments. Joumal of Experimental Psychology, 84, 27-34.

WARD, L. M., \& LockHEAD, G. R. (1971). Response processes in absolute judgment. Perception \& Psychophysics, 9, 73-78.

WEBER, D. L., GREen, D. M., \& LuCE, R. D. (1977). Effects of practice and distribution of auditory signals on absolute identification. Perception \& Psychophysics, 22, 223-231.

(Manuscript received July 6, 1987;

revision accepted for publication December 2, 1987.) 